\title{
Research on Online Education Architecture Based on xAPI Technology
}

\author{
Bu Zhaofeng ${ }^{\mathrm{a}, *}$, Du Xiaoming ${ }^{\mathrm{b}}$ Liu Bin $^{\mathrm{c}}$ and Zhu Ning ${ }^{\mathrm{d}}$ \\ Shijiazhuang Campus, Army Engineering University, Shi Jiazhuang, 050003, China \\ axiaozhaoflying@163.com, dduxiaomingoec@163.com, ${ }^{\mathrm{c}}$ liubinoec@163.com, ${ }^{\mathrm{d}}$ zhuningoec@163.co
}

$\mathrm{m}$

Keywords: online education, xAPI, learning management system, learning record storage.

\begin{abstract}
The current online education platform focuses on providing services to learners, neglecting to track and evaluate learners, not enough to reflect learners' real learning situations and to make accurate evaluations of learning outcomes. Based on the status quo of online education technology and the principle analysis of xAPI technology, three schemes of constructing LMS-centric learning system architecture and two methods of integrating xAPI technology into developing SCORM course package are proposed. Interface introduction and function integration Set up an online education architecture based on xAPI technology, which provides technical support for obtaining education big data.
\end{abstract}

\section{Introduction}

Under the background of informationization, online education and becoming an important form of teaching and training, compared with the traditional classroom teaching, it can rely on the network to learn anytime and anywhere, breaking the shackles of time and space. The current teaching resources are gradually digitized and applied to various online education platforms. Learning Management System (LMS) conforming to the Sharable Content Object Reference Model (SCORM) The teaching environment is further normalized and standardized. As an important means of digital teaching, the problem of reuse and sharing of teaching resources has been solved, and the compatibility and function of teaching platforms have been universally realized. The durability of network teaching has been enhanced. To truly reflect the learner's learning needs a lot of learning data tracking, storage and analysis, Experience API (XAPI) ${ }^{[1]}$ to provide technical support to solve this problem, but also for the development of online education to provide technical support.

\section{Online Education Development}

With the advancement of educational informationization, Massive Open Online Courses (MOOCs) and Small Private Online Courses (SPOCs) have developed rapidly. Online education has spawned teaching methods, Teaching reform.However, the development of online education is inseparable from the support of technical standards. At present, SCORM, as the most widely used E-Learning standard, realizes digital learning functions such as interoperability, reusability and sharing of online learning objects and improves the commonality of teaching resources Sex and teaching platform compatibility $^{[2]}$. As users' demands for online education and training continue to improve, SCORM has revealed some flaws, such as the inability to follow and analyze diverse learning behaviors in a big data education environment. To this end, the Advanced Distributed Learning (ADL) organization in the United States proposed a technology route from SCORM to the Training \& Learning Architecture (TLA) ${ }^{[3]}$.

At present, the online education in China is still in the stage of SCORM or SCORM. The LMS Centric phase takes the "two-track" idea of centering the LMS with the SCORM standard on the learning architecture but incorporating XAPI into the SCORM curriculum to enhance the ability to track learning experiences. The inclusion of the xAPI standard provides technical assurance that a 
learner's learning can be evaluated in a comprehensive manner in order to capture, store, transmit, and share learner experience data.

\section{3. xAPI Principle Analysis}

\subsection{LRS}

The XAPI standard defines LRS as a "repository of learning records" and is primarily responsible for receiving, storing, and providing learning records. Prior to the LRS, LMS was mainly based on the LMS, but LMS can not keep track of learning behavior. While LRS is a new cloud-based storage mechanism with the birth of XAPI, the xAPI standard allows LRS to store almost any learning record for more comprehensive reporting and learner accuracy. LRS only deals with the storage and retrieval of learning experiences, not including the diverse functions of LMS. The "lightweight" feature of LRS greatly reduces its own complexity ${ }^{[4]}$.

\subsection{Statement}

The most important object in the XAPI data model is the "Statement" object, which defines 11 top-level properties such as the Statement data model "Actor", "Verb", "Object", "Result", and "Context" How to express the grammar rules. In Statement, the order of the properties is variable, but the property should not appear more than once in the object, and each statement must include an actor, verb, and object properties. Statement is created in JSON data format and stored in LRS. LRS captures learning experience data by parsing JSON.

\section{3 xAPI works}

XAPI is an application of "activity flow" to tracking data and provides sub-APIs to access and store information about status and content. When a learning activity or learner's behavior needs to be tracked, xAPI issues a statement in the form of "actor + verb + object" (ie "subject + predicate + object"), passing the trace in JSON To LRS, and LRS stores the learning experience recorded as a Statement in the same way as a database ${ }^{[5]}$. LRS can share data, transfer learner's data from one LRS to another LRS or LRS integrated LRS, while LRS with data analysis can generate reports and provide reports of activities for learners.

\section{Based on xAPI Online Education Architecture}

\subsection{SCORM courses integrate XAPI methods}

The SCORM course package is usually a type of ZIP file called the Package Interchange File (PIF). SCORM curriculum package mainly by the manifest file (imsmanifest.xml) and content (content) of two parts. The manifest file is an Extensible Markup Language (XML) file that describes everything in the PIF that contains SCO and assets, and must exist in the root directory. The content includes shareable content objects (SCOs), assets, and supporting files for other SCORMs, where SCO is the instruction unit that contains one or more files that communicate with the LMS and asset is presented to the student but not to the LMS A collection of one or more static content files ${ }^{[6]}$. There are two ways to incorporate XAPI technology into SCORM content packages:

A. Use curriculum generation software to add xAPI statements and related code to SCORM courses. Currently, some curriculum generation software that supports the XAPI standard can choose the activities to be tracked by default, and the course generation software will automatically create and include the tracking information in the PIF by selecting corresponding tracking options.

B. Manually add xAPI-related code to published SCORM course packages. By manually creating a JavaScript file or XAPI statement code and adding its input to the SCO courseware package's SCO, SCO can be identified by looking at the imsmanifest.xml file under the SCORM package root directory, and each SCO will be an XAPI statement track activity".

\subsection{SCORM course integrates the XAPI process}

To technically integrate SCORM with xAPI, you need to understand the SCORM course and LMS communication methods, namely Launch, API, and Data Model. xAPI tracking learner's learning 
experience, we must achieve or control SCORM and LMS communication process, that is, to start and initialize the learning object, support SCORM time model, mapping SCORM data model.

A. Start and initialize learning objects. LMS starts and initializes learning objects with the doInitialize () (SCORM 2004). To control LMS startup and initial learning objects with xAPI, you must implement an "xapi" object so that learning experience data can be passed through an xAPI statement. LMS sets the active configuration information for each startup object by initializing the xAPI instance with initializeAttempt (), setting the status of the SCO, configuring LRS, and so on.

B. Support SCORM time model. In the process of learning object operation, LMS through doGetValue () to get the data information of the active object, stored by doSetValue (), call doTerminate () to terminate the activity. And xAPI needs to function in the process of LMS doSetValue (), call saveDataValue () to handle the type of interaction and the state of the activity, and terminate the activity by calling terminateAttempt ().

C. Map SCORM data model. The data model is the activity interaction information. The LMS stores the corresponding activity data through the data model elements, and the corresponding activity data is described differently in the XAPI. Therefore, the data model elements of the LMS need to be mapped equivalently to the xAPI in order to be converted into xAPI statements, Passed to LRS.

Technically, xAPI was written into the SCORM course package and simply replaced SCORM's data communications protocol and data model. To establish an LRS connection, you must also configure xAPI-related properties in the SCORM course package run-time environment wrapper, including information such as LRS endpoints and LRS authentication.

\subsection{LMS integrated LRS strategy}

\section{A. Free-Standing Program}

The standalone solution is LMS and LRS as two separate systems. While users interact with the LMS, the user's behavior is simultaneously sent to the LRS as a data source. The two work independently and do not affect each other. Learner and LRS information transmission, LMS feedback to the client, and then passed to the client through the LRS, it can be said that LMS and LRS are relatively independent operation, but also indirectly related. This process requires the client to send a request, and the LMS responds and passes data to LRS over HTTP. Learners acquire data from LMS, upload it to LRS data end, acquire learner's behavioral data through import, and use LRS's data analysis engine to construct learner's learning situation. The advantage of this solution is that LMS and LRS operate independently and do not interfere with each other, without considering the technical integration of the two, but the degree of intelligence is not high.

\section{B. Integrated Solution}

The integrated solution realizes the connection with the LRS through the external interface built in the LMS. The user relies on the LMS for the course learning, and the learning experience data is transmitted to the LRS cloud through the network. When establishing a connection to the LRS, the available LRS accounts must be satisfied and the appropriate configuration completed. The advantage of this solution is that the user does not need to manually upload learning experience data to the LRS, and automatically sends the LRS to the LRS through the connection between the LMS and the LRS, thereby improving the data transmission efficiency. However, the data transmission on the network is greatly affected by external influences Poor safety and reliability.

\section{Embedded Solutions}

The embedded solution incorporates LRS functionality into LMS and LRS serves the user as a functional component of LMS. A comprehensive database of LMS and LRS needs to be established for the storage and management of learner's learning experience data, and LRS for learning and experience data retrieval and retrieval. The advantage of this solution is that the learner's learning experience data can be directly tracked, stored and analyzed by the LRS to generate a report, but the technical implementation is difficult. 


\section{Conclusion}

Big data era, online education and learning experience tracking, storage and learning assessment, the report needs the support of big data. XAPI tracks and documents each learner's or group's learning experience in real time and can rely on LRS's data analysis capabilities to process and analyze learning data and generate reports that provide users with real and reliable data for better Master and understand the learner's learning situation. Through the integration with SCORM, it solves the limitations of SCORM curriculum in digital learning and expands SCORM's business capability. Through the application of LRS, it solves the problem that LMS tracks, records, stores, analyzes and shares educational data, and enhances LMS Learning assessment function. The use of xAPI infuses the current SCORM-based digital teaching environment, enabling SCORM-based courses to provide longer-lasting service to learners and driving online education to deeper and broader stages.

\section{References}

[1] ADL.xAPI-Spec Version 1.0.3 [EB/OL]. http://github.com/adlnet/xAPI-Spec/. 2017.

[2] Clayton C. MacAloney. Integration of Experience API into CDET's E-Learning[R]. Naval Postgraduateschool.2016.

[3] Advanced Distributed Learning (ADL) Initiative. Training and Learning Architecture (TLA) [EB/OL].http://www.adlnet.gov/tla.2017.

[4] Peter Berking.Choosing a Learning Record Store Version1.10[S]. Advanced Distributed Learning (ADL) Initiative.2016.

[5] Jonathan M.Kevan,Paul R.Ryan.Experience API:Flexible,Decentralized and Activity-Centric Data Collection[J].Technology Knowledge \& Learning.2016(1):143-149.

[6] Poltrack, J., \& Creighton, T.. Opening legacy data silos: Using experience data for educational impact. Paper presented at the Interservice/Industry Training, Simulation, and Education Conference (I/ITSEC) 2015. 